\title{
Dauer Formation in C. elegans Is Modulated through AWC and ASI-Dependent Chemosensation
}

\author{
PPratima Pandey, ${ }^{1}$ Umer S. Bhat, ${ }^{1}$ Anuradha Singh, ${ }^{1}$ Aiswarya Joy, ${ }^{1}$ Varun Birari, ${ }^{1}$ Nagesh Y. Kadam, ${ }^{1}$ \\ and ${ }^{-}$Kavita Babu ${ }^{1,2}$
}

https://doi.org/10.1523/ENEURO.0473-20.2021

${ }^{1}$ Department of Biological Sciences, Indian Institute of Science Education and Research (IISER) Mohali, Manauli, Punjab 140306, India and ${ }^{2}$ Centre for Neuroscience, Indian Institute of Science, Bangalore, Karnataka 560012, India

\begin{abstract}
The perception of our surrounding environment is an amalgamation of stimuli detected by sensory neurons. In Caenorhabditis elegans, olfaction is an essential behavior that determines various behavioral functions such as locomotion, feeding and development. Sensory olfactory cues also initiate downstream neuroendocrine signaling that controls aging, learning, development and reproduction. Innate sensory preferences toward odors (food, pathogens) and reproductive pheromones are modulated by 11 pairs of amphid chemosensory neurons in the head region of $C$. elegans. Amongst these sensory neurons, the ASI neuron has neuroendocrine functions and secretes neuropeptides, insulin-like peptide (DAF-28) and the TGF- $\beta$ protein, DAF-7. Its expression levels are modulated by the presence of food (increased levels) and population density (decreased levels). A recent study has shown that EXP-1, an excitatory GABA receptor regulates DAF-7/TGF- $\beta$ levels and participates in DAF-7/TGF- $\beta$-mediated behaviors such as aggregation and bordering. Here, we show that exp-1 mutants show defective responses toward AWC-sensed attractive odors in a non-autonomous manner through ASI neurons. Our dauer experiments reveal that in daf-7 mutants, ASI expressed EXP-1 and STR-2 (a G-protein-coupled receptor; GPCR) that partially maintained reproductive growth of animals. Further, studies suggest that neuronal connections between ASI and AWC neurons are allowed at least partially through ASI secreted DAF-7 or through alternate TGF- $\beta$ pathway/s regulated by EXP-1 and STR-2. Together, our behavioral, genetic and imaging experiments propose that EXP-1 and STR-2 integrate food cues and allow the animals to display DAF-7/TGF- $\beta$ neuroendocrine dependent or independent behavioral responses contributing to chemosensensory and developmental plasticity.
\end{abstract}

Key words: ASI neuron; C. elegans; chemotaxis; dauer; EXP-1; STR-2

\section{Significance Statement}

This work sheds light on a possible developmental and postdevelopmental function for the excitatory GABA receptor, EXP-1. We show that mutants of exp-1 are defective in their response toward AWC-sensed odors. Our genetic, behavioral and expression studies reveal that EXP-1 functions in the ASI neuron to modulate chemosensation and to regulate the behavioral switch between dauer and the reproductive state. EXP-1 has been shown to function in a DAF-7/TGF- $\beta$-dependent manner. However, in the absence of DAF-7/ TGF- $\beta$, EXP-1, and a G-protein-coupled receptor (GPCR), STR-2 integrate sensory information to maintain the reproductive state of the animal through an ASI-dependent alternate pathway.

Received November 4, 2020; accepted March 6, 2021; First published March 11, 2021.

The authors declare no competing financial interests.
Author contributions: P.P. designed research; P.P., U.S.B., A.S., A.J., and V.B. performed research; P.P. and N.Y.K. contributed unpublished reagents/ analytic tools; P.P. and K.B. analyzed data; P.P. and K.B. wrote the paper. 


\section{Introduction}

Caenorhabditis elegans inhabit a continually changing environment and thus require very efficient sensory systems to perceive their surroundings for better survival. These animals largely rely on four major sensory systems: taste, touch, temperature and smell (Bargmann, 1998). C. elegans have highly evolved chemosensory abilities as this allows them to survive in unpredictable natural conditions to locate food and sense danger. As soon as the animal hatches into the first instar larval stage, its chemosensory abilities are fully functional and decides its future larval development (Golden and Riddle, 1984a). Sustainable food and growth conditions promote the reproductive cycle, whereas food scarcity and increased pheromone levels because of overcrowding promote entry into the dauer stage (Cassada and Russell, 1975; Golden and Riddle, 1984a,b).

Chemosensory signals are perceived through gustatory (water-soluble cues) or olfactory (volatile cues) amphid neurons (Bargmann et al., 1993). Olfactory cues are recognized from greater distances when compared with the gustatory cues (Bargmann and Mori, 1997). The AWC and AWA neurons in C. elegans detect volatile attractants whereas the AWB neuron is responsible for sensing aversive odors (Bargmann and Horvitz, 1991). Sensory neurons are in direct contact with the environment to perceive external cues, initiate neuroendocrine signaling that modulate vital developmental decisions and behaviors (Nowels and Thomas, 1994; Coburn and Bargmann, 1996; Daniels et al., 2000). To sense the environment very precisely with limited neurons, chemosensory neurons in C. elegans have evolved to express multiple receptors in contrast to mammalian neurons where each neuron harbor a single set of receptors (for review, see Hart and Chao, 2010). Spatial and temporal expression of many chemoreceptors is regulated by the DAF-7/TGF- $\beta$ pathway (Zwaal et al., 1997; Birnby et al., 2000; Daniels et al., 2000; Sengupta, 2007). DAF-7/TGF- $\beta$ is secreted from the ASI chemosensory neurons (amphid neurons) in response to environmental signals such as pheromones, pathogens, temperature and food.

This work was supported by the Department of Biotechnology (DBT)Wellcome Trust India Alliance (IA) Grants IA/I/12/1/500516 and IA/S/19/2/ 504649 (to K.B.) and partially supported by DBT Grant BT/PR24038/BRB/10/ 1693/2018, Ministry of Human Resources Development (MHRD)-Scheme for Transformational and Advanced Research in Sciences (STARS) Grant STARS/ APR2019/BS/454/FS, and Department of Science and Technology (DST)Science and Engineering Research Board (SERB) Grant SERB/F/7047 as well as a DBT-IISc partnership grant and IISER Mohali core funding (K.B.). P.P. is supported by the DST WOS-A Grant SR/WOS-A/LS-285/2018 and was supported by the DBT Bio-CARe Grant BioCARe/01/10167. The mutant strains were procured from CGC, which is funded by the National Institutes of Health Office of Research Infrastructure Programs Grant P40 OD010440.

Acknowledgements: We thank Ankit Negi for routine help, Anupreet Saini for help with genotyping and chemotaxis assays, and the Indian Institute of Science Education and Research Mohali Confocal facility for use of the confocal microscope.

Correspondence should be addressed to Pratima Pandey at pratimashpandey@gmail.com or Kavita Babu at kavita.babu@babulab.org.

https://doi.org/10.1523/ENEURO.0473-20.2021 Copyright @ 2021 Pandey et al.

This is an open-access article distributed under the terms of the Creative Commons Attribution 4.0 International license, which permits unrestricted use, distribution and reproduction in any medium provided that the original work is properly attributed.
Defects in DAF-7/TGF- $\beta$ signaling result in phenotypes such as constitutive dauer formation, increased life-span, aggregation and bordering (Thomas et al., 1993; Peckol et al., 2001; Cheung et al., 2004; Shaw et al., 2007). The levels of DAF-7/ TGF- $\beta$ were shown to be regulated by a GABA receptor, EXP-1 that also participates in aggregation and bordering behaviors seen in daf-7 mutants (Bendesky et al., 2012).

The EXP-1 gene is an excitatory GABA receptor that is expressed in enteric muscles of the intestine, where it mediates muscle contractions in response to GABA signaling from motor neurons (Beg and Jorgensen, 2003). EXP-1 is also expressed in five sets of head neurons (SABD, RID, $A D E$, and two unidentified neurons; Beg and Jorgensen, 2003). It has been previously reported that GABA signaling in the accessory olfactory bulb of rats is excitatory in nature (Goldmakher and Moss, 2000). This led us to investigate the role of EXP-1 in chemotaxis/olfaction. Mutants of exp-1 when tested in chemotaxis assays were specifically defective toward AWC-sensed odors. At the molecular level, neurons sense cues through G-proteincoupled receptors (GPCRs) present on their surface (Troemel et al., 1995; Sengupta et al., 1996). The expression of multiple olfactory GPCRs in the ASI chemosensory neurons (SRA-6, STR-2, STR-3, STR-1, SRD-1) is modified on exposure to pheromones and during dauer stages of development through DAF-7/TGF- $\beta$ (Nolan et al., 2002; Lans and Jansen, 2006). The STR-2 receptor normally expressed in the AWC neuron is upregulated in the ASI neuron during dauer stages or in the absence of DAF7/TGF- $\beta$ signaling (Peckol et al., 2001). Transition in receptor levels affect neuron morphology and alter behaviors (Peckol et al., 2001; Dixit et al., 2020). To investigate the receptor-dependent developmental plasticity of the organism, we studied the role of STR-2 and EXP-1 in dauer formation. We show that STR-2 and EXP-1 in the ASI neuron sense the availability of food and promote reproductive stages in the absence of DAF-7/TGF- $\beta$. Further, simultaneous loss of str-2 and exp-1 in the daf-7 mutant background progressed the animals into reproduction defective dauer stages.

\section{Materials and Methods}

\section{C. elegans strains and maintenance}

All C. elegans strains were maintained at $20^{\circ} \mathrm{C}$ on nematode growth media (NGM) plates seeded with Escherichia coli OP50 (Brenner, 1974). The strains used in this study are compiled in Table 1. All the strains were outcrossed at least three times. The exp-1 mutant strain EG276 was outcrossed frequently (once every three to four months). N2 Bristol was used as the wild-type (WT) reference strain. Double or triple mutants were generated by standard genetic crosses and verified by PCR or fluorescence expression. The primers used for genotyping during outcrossing and for the generation of double or triple mutants are listed in Table 2.

\section{Plasmid construction and germline transformation}

Constructs used in this study were generated by standard molecular biological techniques (Russell, 2001). Plasmids used to generate the rescue constructs were 
Table 1: List of strains used in this study

\begin{tabular}{|c|c|c|}
\hline Strain & Genotype & Comments \\
\hline EG276 & $\exp -1(o x 276) / I$ & $3 \times$ out crossed (CGC strain) \\
\hline RB2302 & Daf-7(ok3125)III & $3 \times$ out crossed (CGC strain) \\
\hline VC3044 & $d b /-1(o k 3749) V$ & $3 \times$ out crossed (CGC strain) \\
\hline VC2413 & str-2(ok3089) V & $3 \times$ out crossed (CGC strain) \\
\hline RB2464 & $\operatorname{tax}-2(0 k 3403) !$ & $3 \times$ out crossed (CGC strain) \\
\hline VC3113 & tax-4(ok3771) III & $3 \times$ out crossed (CGC strain) \\
\hline FK311 & ceh-36(ks86) X & $3 \times$ out crossed (CGC strain) \\
\hline FK181 & ksls2[Pdaf-7::GFP + rol-6(su1006)] & $3 \times$ out crossed (CGC strain) \\
\hline CX3695 & Pstr-2::GFP(kyls140) & CGC strain \\
\hline BAB1415 & exp-1(ox276); str-2 (ok3089) & This study \\
\hline BAB1421 & exp-1(ox276); daf-7(ok3125); str-2(ok3089) & This study \\
\hline BAB1424 & exp-1(ox276); daf-7(ok3125) & This study \\
\hline BAB0881 & exp-1(ox276); ceh-36(ks86) & This study \\
\hline BAB0882 & tax-2(ok3403; exp-1(ox276) & This study \\
\hline BAB0880 & $\exp -1(o x 276) ; \operatorname{tax}-4(o k 3771)$ & This study \\
\hline BAB1577 & daf-7(ok3125); str-2(ok3089) & This study \\
\hline BAB1588 & exp-1/Pexp-1::EXP-1in pPD95.75 (indEx892) & This study \\
\hline BAB1589 & WT/Pexp-1::EXP-1::s|2::wrmScarlet (indEx893) & This study \\
\hline BAB1590 & exp-1/Pexp-1::EXP-1::sl2::wrmScarlet (indEx894) & This study \\
\hline BAB1591 & exp-1/ Pgpa-4::EXP-1::s|2::wrmScarlet (indEx895) & This study \\
\hline BAB1592 & (indEx894)/Psrb-6::GFP (indEx896) & This study \\
\hline BAB1593 & exp-1;str-2/Pgpa-4::STR-2::sl2::wrmScarlet (indEx897) & This study \\
\hline BAB1594 & exp-1;str-2/Pstr-2::STR-2::sl2::wrmScarlet (indEx898) & This study \\
\hline BAB1595 & kyls140/Pdaf-1::sl2::wrmScarlet (indEx899) & This study \\
\hline
\end{tabular}

Table 2: List of oligonucleotides used in this study

\begin{tabular}{|c|c|c|c|}
\hline Primer code & Sequence & Comment & Gene \\
\hline PRS 40 & CGT GGC GAG ACC CTC GAC & Forward external & $\exp -1$ \\
\hline PRS 41 & CTC GAA CGT AGC CGC CAA TTC & Forward internal & $\exp -1$ \\
\hline PRS 42 & GTG CCA ACT TTA TCA GGG AGA G & Reverse external & $\exp -1$ \\
\hline PRS 439 & AGGACGGAAATTACCTGTGC & Forward external & daf-7 \\
\hline PRS 440 & GCTTCGGGAAACGCTCATAT & Reverse external & daf-7 \\
\hline PRS 441 & TTATTCTTCTTGTCGGGGCC & Reverse internal & daf-7 \\
\hline PRS 362 & CTATTGCTTGCGCTCATAGGAAG & Genotyping forward external & str-2 \\
\hline PRS 363 & CTTCAGTCATCATGTTGCACAATTTC & Genotyping reverse external & str-2 \\
\hline PRS 364 & GTGTAATGTGGATTATACTTGTCAG & Genotyping reverse external & str-2 \\
\hline PRS 426 & AGCTGTTGTCTCCTTCCAGG & Genotyping forward WT & $\operatorname{tax}-2$ \\
\hline PRS 427 & CGATTTCCGATGAGGAAACC & Genotyping forward mutant & $\operatorname{tax}-2$ \\
\hline PRS 428 & CACAGCTTCTAATAGGAAAGGG & Genotyping reverse Common & $\operatorname{tax}-2$ \\
\hline PRS 394 & GCGGTTCGGATACGAAAATACTTG & Genotyping forward external & $\operatorname{tax}-4$ \\
\hline PRS 395 & GACGGAGAAGTGTATCCGTTATATC & Genotyping reverse internal & $\operatorname{tax}-4$ \\
\hline PRS 396 & CCATGCGTCCGTCCCTAATCC & Genotyping reverse external & $\operatorname{tax}-4$ \\
\hline PRS 403 & GTGTGGTACCCAAGTTGATAGG & Genotyping forward common & ceh-36 \\
\hline PRS 404 & GTTTTCCGCGAAACACAGTACC & Genotyping reverse WT & ceh-36 \\
\hline PRS 405 & GTTTTCCGCGAAACACAGTACT & Genotyping reverse M & ceh-36 \\
\hline PRS 576 & GGAGAGTCGTCATCGGCG & Genotyping forward external & $d b /-1$ \\
\hline PRS 577 & GGCATTGGATTTGGACAAGAGC & Genotyping reverse external & $d b /-1$ \\
\hline PRS 578 & CTGTGCAGACTGGTCCGAG & Genotyping forward internal & $d b /-1$ \\
\hline PRS 349 & AAAACTGCAGcttgagagatccaatgaaatcgg & Cloning forward Pstl & exp-1 promoter in pPD95.75 \\
\hline PRS 351 & CTAGTCTAGAgccatcaagttttggcag & Cloning reverse $\mathrm{Xbal}$ & exp-1 promoter in pPD95.75 \\
\hline PRS 352 & GGGGTACCatgtctgcatctattctaattttg & Cloning forward Kpnl & exp-I Genomic in pPD95.75 \\
\hline PRS 353 & CCGCTCGAGctagtagatgtcggcaaaccactc & Cloning reverse $\mathrm{Xhol}$ & exp-I Genomic in pPD95.75 \\
\hline PRS 369 & ACATGCATGCgaacggtctgtgggctctgac & Cloning forward Sphl & str-2 promoter \\
\hline PRS 370 & AAAACTGCAGgcagacccatatgtgtgcacaaac & Cloning reverse Pstl & str-2 promoter \\
\hline PRS 515 & ACATGCATGCgaagccttgtttgtataagaaacgctg & Cloning forward Sphl & gpa-4 promoter \\
\hline PRS516 & CCCCCCGGGGttgaaaagtgttcacaaaatgaataagtg & Cloning reverse $\mathrm{Xmal}$ & gpa-4 promoter \\
\hline PRS 561 & CACTTTTCAACGGatccccgggatgccgactgtgcaatgg & Cloning forward Xmal & str-2 genomic in a wrmScarlet vector \\
\hline PRS 566 & GGGGTACCgcttgcgctcataggaagag & Cloning reverse Kpnl & str-2 genomic in a wrmScarlet vector \\
\hline PRS 534 & ACATGCATGCcggtcaccattcccaaattcgc & Cloning forward Sphl & daf-1 promoter \\
\hline PRS 535 & GGGGTACCcttgcacaggtaccaatttatgatg & Cloning reverse Kpnl & daf- 1 promoter \\
\hline
\end{tabular}


Table 3: List of plasmids used in this study

\begin{tabular}{lll}
\hline S. number & Plasmid number & Plasmid \\
\hline 1 & pBAB0056 & Pexp-1::EXP-1in pPD95.75 \\
2 & pBAB0063 & Pexp-1::EXP-1::sl2::wrmScarlet \\
3. & pBAB0067 & Pgpa-4::EXP-1::sl2:::wrmScarlet \\
4. & pBAB0069 & Pgpa-4::STR-2::sl2::wrmScarlet \\
5. & pBAB0070 & Pstr-2::STR-2::sl2:::wrmScarlet \\
6. & pBAB0071 & Pdaf-1::sl2:: wrmScarlet \\
7. & pBAB465 & Psrb-6::GFP (Kadam et al., 2021)
\end{tabular}

pPD95.75, pPD49.26 or the wrmScarlet vector (Prig-3:: NPR-4::s/2::wrmScarlet) that was kindly provided by the Cori Bargmann lab. Germline transformation of the constructs was performed by microinjection as previously described (Mello et al., 1991; Mello and Fire, 1995). The promoter fusion constructs for expression and gene rescuing constructs were injected at $20-30 \mathrm{ng} / \mu \mathrm{l}$. The constructs used in this study are tabulated in Table 3.

\section{Chemotaxis assay}

To perform Chemotaxis assays, eggs were prepared and on hatching the $C$. elegans were grown till young adult stages. Assay was performed after $65 \mathrm{~h}$ (h) of incubation at $20^{\circ} \mathrm{C}$. NGM plates w/o cholesterol were used for the assay. Wherever required, odorants were diluted in ethanol and reported as percent by volume. Modified $90 \mathrm{~mm}$ quadrant plate chemotaxis assays were performed as described previously (Bargmann et al., 1993; Margie et al., 2013). Plates were prepared a day in advance and dried for $1 \mathrm{~h}$. Animals were collected using M9 buffer with $0.003 \%$ Tween 20 and washed three times with M9 buffer. Plates were divided into four quadrants and marked as control (C) and test (T) spots in adjacent sections and $3 \mathrm{~cm}$ from the loading center. To these spots, $2.0 \mu \mathrm{l}$ of freshly prepared $1 \mathrm{~m}$ sodium azide (in M9) was added. Sodium azide acts as an anesthetic agent to immobilize $C$. elegans that reach the vicinity of the spot during the assay. After $2 \mathrm{~min}$, animals (200-250) were added in the center of the plate. Once the $C$. elegans were ready on the assay plate, we added ethanol $(3.0 \mu l)$ to the control spots and odors $(3.0 \mu l)$ to the test spots. Plates were incubated for 90 min undisturbed and immediately after that moved to a $4^{\circ}$ $\mathrm{C}$ refrigerator. Animals in the respective quadrants were counted after 3-4 h, and chemotaxis indices were calculated using the previously published formula; chemotaxis index = (number of animals in both test quadrants - number of animals in both control quadrants)/(total number of animals scored; Margie et al., 2013). Graphs of chemotaxis indices were then plotted. In case where animals were dauer, to get rid of dauer animals while washing, the adult animals were allowed to settle down and dauers were removed from the supernatant during a washing step. We counted only the $C$. elegans that moved from the center of the plate. WT animals showed a chemotaxis index of $\sim 0.7$ for attractive odors and animals with chemotaxis defects showed lower or higher values.

\section{Dauer assay}

Freshly prepared eggs were transferred to 60-mm NGM plates. Each plate was plated with $\sim 250-300$ eggs.
Plates were incubated at upto three different temperatures; $16^{\circ} \mathrm{C}, 20^{\circ} \mathrm{C}$, and $25^{\circ} \mathrm{C}$. Animals were allowed to grow, and once the $C$. elegans reached the adult stage, the animals were counted. Animals took variable times to grow at different temperatures. The timeline that was followed for scoring were $\sim 60 \mathrm{~h}$ for $25^{\circ} \mathrm{C}, \sim 70 \mathrm{~h}$ for $20^{\circ} \mathrm{C}$ plates, and the $16^{\circ} \mathrm{C}$ plates were scored for dauer animals after $92 \mathrm{~h}$ (Ailion and Thomas, 2000). Graphs were plotted for the dauer phenotype as the percentage of dauers in the total population of $C$. elegans.

\section{Microscopy}

Fluorescence imaging was performed on live animals. Briefly, animals were immobilized using $30 \mathrm{mg} / \mathrm{ml}$ 2,3-butanedione monoxamine (Sigma-Aldrich) prepared in M9 as previously described (Sieburth et al., 2005). In order to visualize and image, the animals were immobilized with $30 \mathrm{mg} / \mathrm{ml} \mathrm{BDM}$ on $2 \%$ agarose pads (prepared in M9); $1 \mu \mathrm{m}$ (total depth) Z-series stacks were collected using a Carl Zeiss fluorescence microscope Axio Imager Z2 with the Axiocam MRm camera equipped with GFP and red fluorescent protein filters. Images were collected and analyzed using the FIJI image J software (Schindelin et al., 2012). Maximum intensity projections of Z-series stacks were used for the analyses of expression and localization of fluorescent markers. The exposure settings were kept identical for all images taken in a single experiment. Imaging was done in the head region of the animals and 25-30 animals were imaged for each experiment. To image AWC and ASI cilia, imaging was performed using the Leica TCS SP8 confocal microscope, using the argon laser at $10 \%$ gain.

\section{Tracking of C. elegans}

We recorded the chemotaxis behavior of animals for 90 min using a 5-megapixel CMOS USB camera (Mightex) and used the Mightex Camera Demo v1.1.0 software. Recordings were done in a Peltier cooled incubator at $20^{\circ}$ $\mathrm{C}$ at $0.5 \mathrm{frames} / \mathrm{s}$ (Dahiya et al., 2019). Tracks of the animals were analyzed using the FIJl software (Schindelin et al., 2012).

\section{Statistical analysis}

To perform statistical analysis, GraphPad Prism version 6.0 was used. The error bars represent SEM calculated for chemotaxis and dauer assay data. Statistical comparisons were done using one-way ANOVA with the Bonferroni multiple comparison test. The level of significance was set as ${ }^{*} p<0.05,{ }^{* \star} p<0.01$, and ${ }^{* \star *} p<0.001$.

\section{Results}

\section{Mutants in exp-1 show defective response toward AWC-sensed odors}

It has been previously reported that EXP-1 is expressed in a few head neurons in addition to its expression in the enteric muscles of the intestine (Beg and Jorgensen, 2003). There were a pair of unidentified neurons that expressed EXP-1, and we were interested in delineating 
A

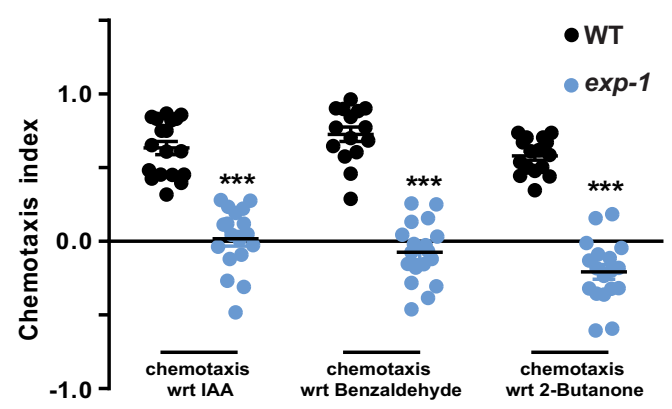

C

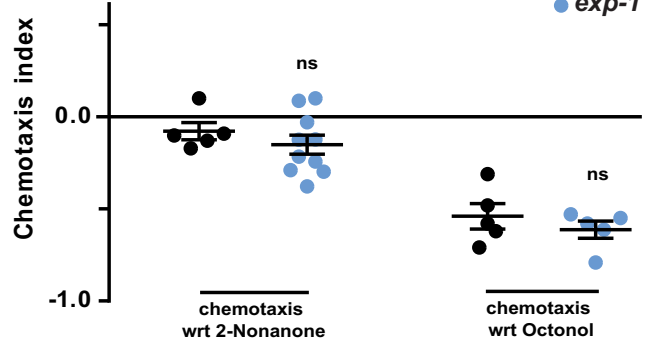

E
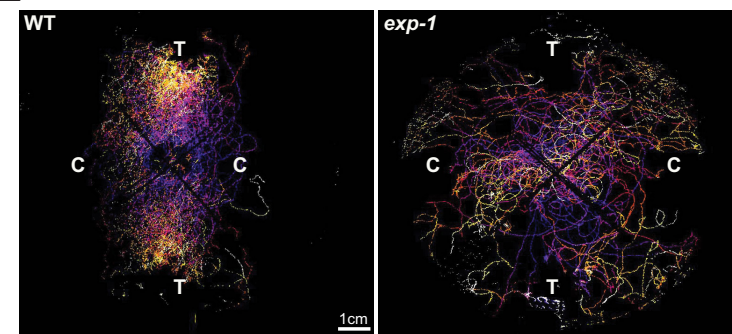

B

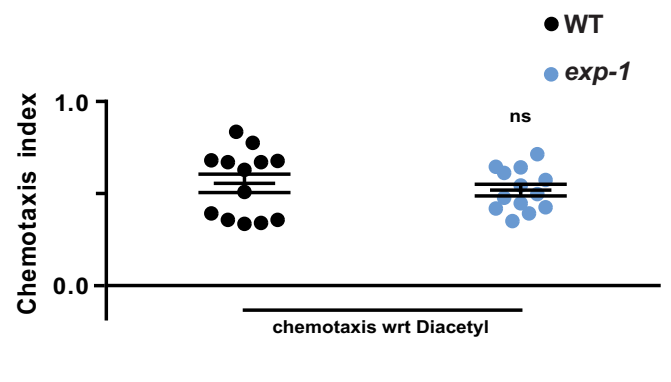

D

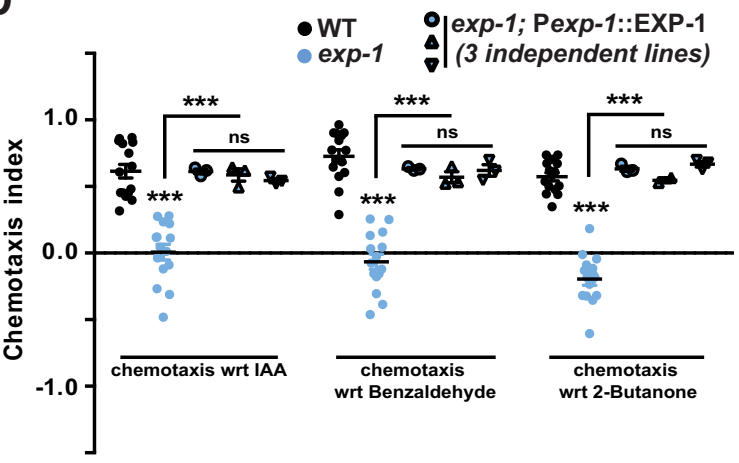

$\mathbf{F}$
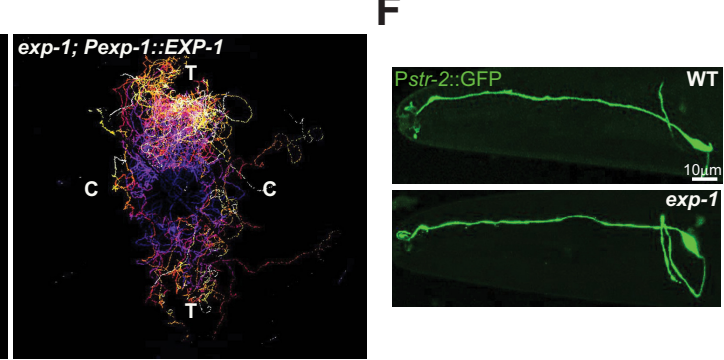

Figure 1. exp-1 mutants show defects in AWC-dependent chemosensation. A, Graph indicating chemotaxis indices for AWCsensed attractants, IAA, benzaldehyde, and 2-butanone used at 1:1000 dilution for WT and exp-1 animals. $\boldsymbol{B}$, Response of WT and exp-1 C. elegans toward the AWA-sensed attractant diacetyl (1:1000). C. Avoidance response of exp-1 and WT animals toward the AWB-sensed odors, Nonanone and 1-octanol (1:100 dilution for both). Each circle in the graphs represents one assay performed using $\sim 200-250$ C. elegans for all graphs from $\boldsymbol{A}-\boldsymbol{C}$. $\boldsymbol{D}$. Defects in exp-1 mutants were rescued using the endogenous exp-1 promoter. Each circle or triangle in the graph represents one assay performed using $\sim 200-250$ C. elegans. E, Tracks of WT (left panel), exp-1 (middle panel), and the exp-1 rescuing line (right panel) animals during chemotaxis toward IAA. F, Fluorescent confocal images of the AWC neuron of WT and exp-1 mutants expressing Pstr-2::GFP. In all graphs, the error bars represent SEM; $p$ values are indicated as ${ }^{* \star *} p<0.001$; ns, not significant in all graphs based on $p$ values calculated using one-way ANOVA with Bonferroni multiple comparison test.

these neurons. The morphologic position of these neurons was evaluated by referencing to Worm Atlas. We suspected the neurons to be AWC neurons. To this end, we tested exp-1 mutant animals for volatile odors sensed by the AWC neurons, using established chemotaxis assays (Bargmann and Horvitz, 1991). First, we tested for isoamyl alcohol (IAA), and found that the exp-1 mutant animals were defective in recognizing this odor (Fig. 1A). To further investigate the olfactory defects of exp-1 mutants we tested these mutant animals for other attractive volatile odors (benzaldehyde, 2-butanone) sensed by the AWC neuron. We found that exp-1 animals were defective in the recognition of all the known attractive odors sensed by the AWC neuron (Fig. 1A). To understand whether this behavior was specific for AWC-sensed volatile odors, we tested exp-1 mutant animals for an AWA-sensed attractive odor, diacetyl and found that these animals behaved like WT control animals in the presence of diacetyl (Fig. $1 B)$. Next, we tested exp-1 mutants against the repulsive volatile odors, nonanone and 2-octanol that are sensed by the AWB neuron. Mutants in exp-1 were indistinguishable from WT animals in their repulsion to nonanone and 2-octanol (Fig. 1C). Our data suggest that exp-1 mutant animals were defective only for AWC-sensed odors. We next generated a rescue construct by using the endogenous exp-1 promoter to express EXP-1. Chemotaxis assays were performed, and we observed that expressing EXP-1 under its native promoter in exp-1 mutant animals 
rescued the chemotaxis defects seen in the mutant $C$. elegans (Fig. 1D). We also wanted to understand the movement of animals during chemotaxis and tracked WT, exp1 mutants, and exp-1 mutants containing the rescue construct during the chemotaxis assay. Our data showed that in case of WT and the exp-1 rescue lines, the animals very quickly oriented in the direction of the attractant and moved to the quadrants containing the attractants (Fig. $1 E$, left and right panels). The exp-1 mutant animals however moved all over the plate and a majority of these C. elegans localized to the control quadrants showing that they were not attracted to AWC-sensed attractants (Fig. $1 E$, middle panel). Previous studies have shown that defects in the AWC cilia, that are the sites of odor perception, can lead to defective responses toward odors (O'Halloran et al., 2009). We speculated that there could be defects in the cilia of AWC neurons in exp-1 mutants, and this might explain the defects toward AWC-sensing odors. We imaged and analyzed the AWC neurons of control and exp-1 mutant animals and found no obvious difference in the morphology of the AWC neurons or their cilia (Fig. 1F). These results suggest that EXP-1 participates in AWC neuron-dependent chemotactic behaviors, although exp-1 mutants show no significant defects in the AWC neuron morphology.

\section{EXP-1 is expressed and functions in the ASI chemosensory neuron}

In order to understand the neuronal basis of exp-1 mutant defects, we studied the expression pattern of EXP-1. To study the expression of EXP-1, we generated a Pexp$1::$ EXP-1::SL-2::wrmScarlet expression line. Upon imaging we observed that as reported previously, there was expression in the enteric muscles and in the head neurons (Fig. 2A; Beg and Jorgensen, 2003). Since we found AWC-dependent phenotypes in exp-1 mutants, we used the AWC-expressing str-2::GFP marker line and studied its co-localization with Pexp-1::EXP-1::s|2::wrmScarlet. Surprisingly, our imaging results revealed that EXP-1 was not expressed in the AWC neurons (Fig. $2 A$, upper panel). Since exp-1 mutants showed aversive behavior toward attractants, we performed EXP-1 expression analysis in the ASH neurons (Troemel et al., 1995; Chao et al., 2004). We crossed an ASH neuron-specific marker line, Psrb-6:: GFP into the Pexp-1::EXP-1::sl-2::wrmScarlet background and studied the localization of the two fluorescent markers. Our imaging analysis suggested that EXP-1 was not expressed in the ASH neurons (Fig. 2A, middle panel). Based on previous studies where EXP-1 was shown to participate in ASI-dependent behaviors (Bendesky et al., 2012), we studied EXP-1 expression in the ASI neurons. ASI neurons are amphid sensory neurons with direct links between the sensory input in the form of food cues and neuroendocrine signaling through the production of DAF7/TGF- $\beta$ (Golden and Riddle, 1982, 1984a; Nolan et al., 2002). For this experiment, a Pdaf-7::GFP marker line was introduced into the EXP-1 expression line. Our imaging results clearly revealed that EXP-1 was localized to the ASI neuron along with Pdaf-7::GFP (Fig. 2A, lower panel). ASI is a chemosensory neuron that belongs to the group of amphid neurons whose cilia are directly exposed to the outer milieu (Bargmann and Horvitz, 1991). It detects water-soluble chemicals and pheromones and is the major regulator of DAF-7/TGF- $\beta$-dependent dauer formation (Albert et al., 1981; Golden and Riddle, 1982, 1984a; Gottlieb and Ruvkun, 1994; Ren et al., 1996; Schackwitz et al., 1996). A previous study has shown that in dauer stages the cilia are retracted (Ward et al., 1975) and retracted cilia have been shown to sense volatile odors (Bargmann et al., 1993). In order to study the cilia in the ASI neurons, we performed imaging analysis of the ASI ciliary endings in WT and exp-1 mutant animals. Confocal imaging was performed and the ciliary endings were measured from the extreme anterior end of animals (illustrated in Fig. $2 B$, left panel). Analyses of exp-1 mutants (Fig. 2B, lower panel) when compared with the WT control animals (Fig. $2 B$, upper panel) revealed that in these mutants, the ASI cilia were significantly withdrawn from the amphid pore (Fig. 2B, bottom panel). Next, we expressed EXP-1 specifically in the ASI neurons using the gpa-4 promoter (Pgpa-4::EXP-1) and found that ASI expression of EXP-1 completely rescued the chemotaxis defects associated with the exp-1 mutants (Fig. 2C). These rescue experiments revealed that the alteration in chemotaxis behavior of exp-1 was also ASI dependent. Together, these data revealed that EXP-1 is expressed and appears to function in the ASI neurons to regulate AWC-dependent chemotaxis.

\section{EXP-1 participates in AWC-dependent chemotaxis in a non-cell autonomous manner}

As detailed above, exp-1 mutants are defective for AWC-sensed odors. We next wanted to examine the role of the AWC neuron in this behavioral defect. First, we asked what would be the response of exp-1 mutants toward odor detection if AWC sensory neurons were not functional. Here, we used a mutant of ceh-36. CEH-36 is an OTX/OTD transcription factor and plays a role in the terminal differentiation of AWC neurons. Loss of ceh-36 results in the loss of chemosensation toward AWCsensed odors (Lanjuin et al., 2003). When we assayed the double mutants of ceh-36; exp-1, it showed defects similar to those seen in ceh-36 mutant animals (Fig. 3A).

Next, we examined the primary AWC chemosensory transduction genes tax-2 and tax-4 that encode cyclic nucleotide gated channels, which transduce signals sent by GPCRs such as the str genes (Coburn and Bargmann, 1996; Komatsu et al., 1999; Cho et al., 2004, 2005). Notably, they are required in AWC neurons for chemosensory signal transduction and mutation in these genes lead to defects in AWC-dependent chemosensory responses (Fig. 3B; Bargmann et al., 1993; Chalasani et al., 2007). We generated double mutants of exp- 1 with both tax-4 and tax-2, respectively. Our results demonstrated that double mutants generated with these signal transduction genes were also defective in chemotaxis, and showed behaviors similar to that seen in the tax- 4 and tax-2 single mutants (Fig. 3B). These results demonstrate that the chemotaxis defects in exp-1 mutants are similar to the defects observed because of defective AWC neurons and 
A

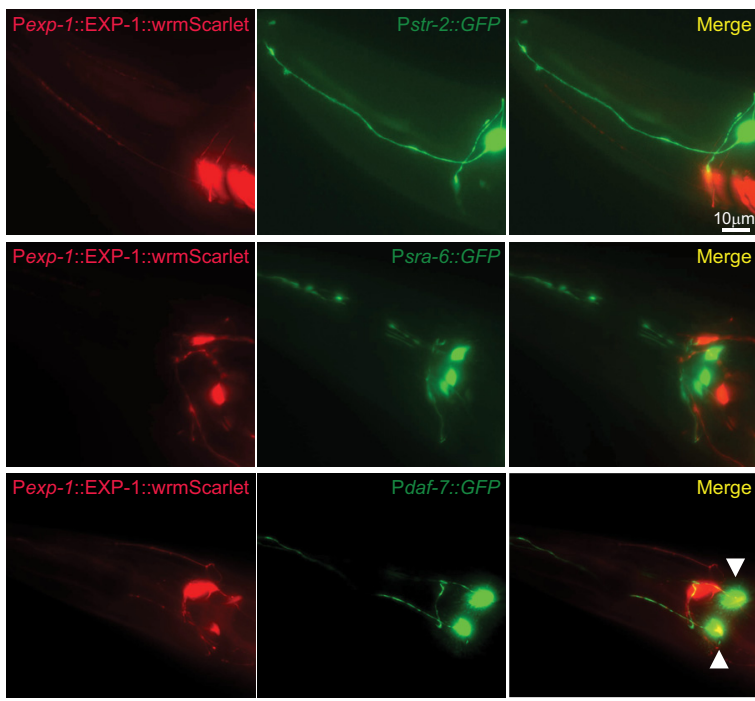

B
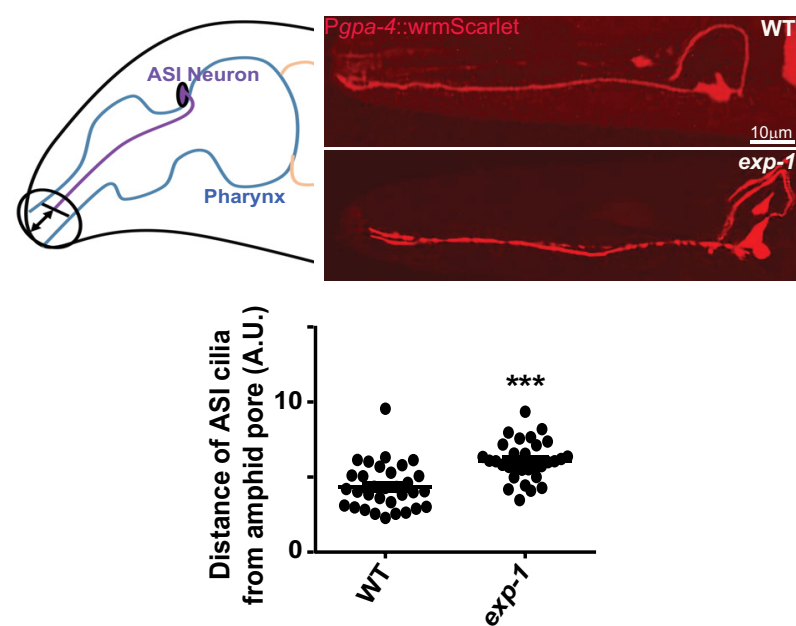

C

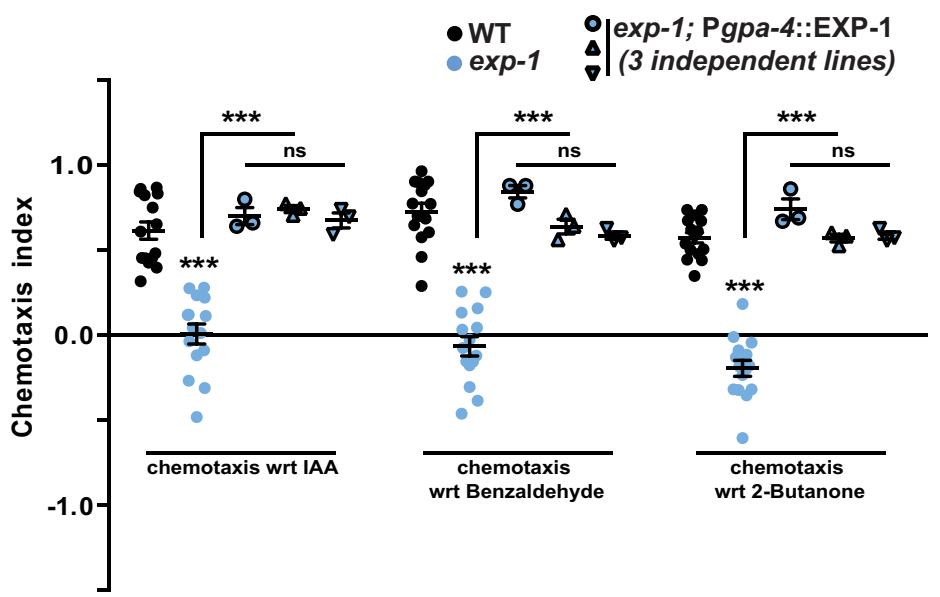

Figure 2. EXP-1 is expressed in the ASI amphid neuron and regulates AWC-dependent chemotaxis in a non-cell autonomous manner. $\boldsymbol{A}$, Localization of Pexp-1::wrmScarlet with respect to the AWC neuron marked with GFP (top panel), ASH neurons marked with GFP (middle panel), and the ASI neurons marked with GFP (bottom panel). B, Illustration of the ciliary region of the ASI neuron toward the amphid pore (left panel). Confocal images of the ciliary region of the ASI neuron from WT (upper right panel) and exp-1 mutants (lower right panel). The bottom panel indicates a graph depicting the distance of the ciliary end from the externally exposed end of the amphid pore as shown in the illustration. This was measured as an arbitrary unit (A.U.) by using the analysis tool of FIJI. Each circle corresponds to one animal. $\boldsymbol{C}$, AWC-dependent chemotaxis defect of exp-1 mutants were rescued by ASI-specific 
continued

expression of EXP-1. Each circle or triangle in the graph represents one assay performed using 200-250 C. elegans. Error bars represent SEM; $p$ values are indicated as ${ }^{\star \star \star} p<0.001$; ns, not significant in all graphs based on $p$ values calculated using one-way ANOVA with Bonferroni multiple comparison test.

A

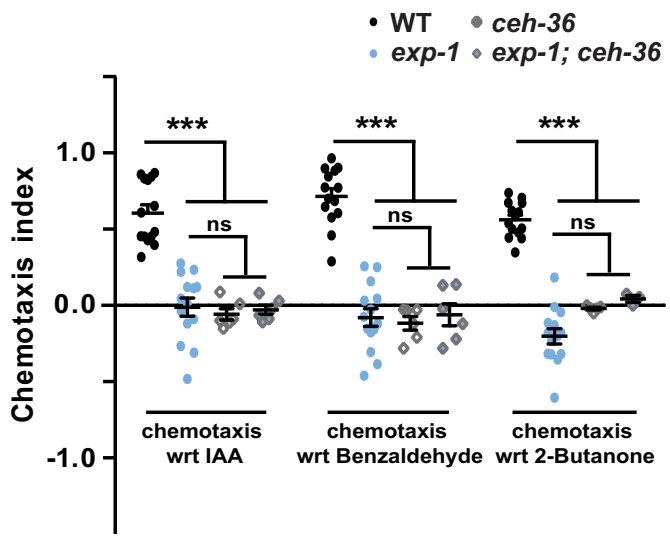

B

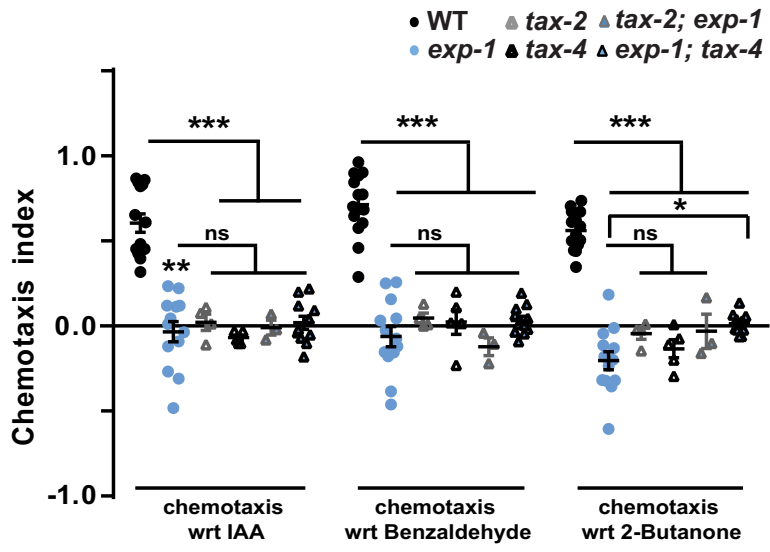

C

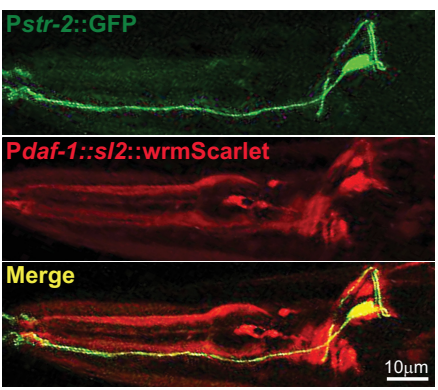

Figure 3. The chemotaxis defects of exp-1 require a functional AWC neuron. $\boldsymbol{A}$, Graph indicating chemotaxis indices of exp-1; ceh-36 C. elegans along with control animals. B, Graph indicating chemotaxis indices of tax-2; exp-1 and exp-1; tax-4 C. elegans along with control animals. Each circle, quadrilateral, or triangle in the graphs represents one assay performed using 200-250 C. elegans. Error bars represent SEM; $p$ values are indicated as ${ }^{* * *} p<0.001,{ }^{* *} p<0.001$, and ${ }^{*} p<0.05$ in all graphs based on $p$ values calculated using one-way ANOVA with Bonferroni multiple comparison test. $\boldsymbol{C}$, Fluorescence images of AWC neuron showing co-expression of STR-2 (top panel) and DAF-1 proteins (middle and bottom panels).
AWC developmental and signaling components did not show additive defects with exp-1 mutant animals. Our data thus far suggest that although EXP-1 is expressed in the ASI chemosensory neurons, it appears to be required for the chemosensory function of the AWC neurons.

To understand the non-cell autonomous function of EXP1 , we wanted to understand the connectivity between ASI and AWC and found that ASI makes chemical synapses with the AWC neuron (Wormatlas; Oshio et al., 2003). The ASI neuron secrete small molecules like neuropeptides, insulin like peptides and/or DAF-7/TGF- $\beta$ that could aid in the cross talk between ASI and AWC. DAF-7 expression was shown to be regulated by EXP-1 (Bendesky et al., 2012), and we hypothesized that it might be through DAF-7 that EXP-1 is regulating AWC function. DAF-7 signals are transduced through its widely expressed receptor components DAF-1 and DAF-4 that function as heterotetramers (Patterson et al., 1997; Gunther et al., 2000; Inoue and Thomas, 2000; da Graca et al., 2004). We speculated that these receptors may be present on AWC neurons which would then receive DAF-7 signals from ASI neurons. We chose to study DAF-1 receptor as DAF-4 also binds with another TGF- $\beta$ protein DBL-1. To study DAF-1 expression in the AWC neuron we transformed DAF-1::WrmSc into the AWC reporter (STR-2::GFP) line and performed imaging experiments. Our results clearly showed that DAF-1 localizes to the AWC neuron (Fig. $3 C$ ). Hence, the AWC neuron could receive DAF-7 signals from the ASI neuron. This hypothesis is also supported by our previous results where we found that expression of EXP-1 in the ASI neuron fully rescued the chemotaxis defect of exp-1 mutants (Fig. $2 B$ ). Thus, EXP-1 appears to be functioning in a non-cell autonomous manner to regulate AWC-mediated chemotaxis toward attractants and the cross talk could be occurring through DAF-7/TGF- $\beta$ signaling.

\section{The chemotaxis defects of exp-1 mutants are mediated through DAF-7/TGF- $\beta$ and STR-2}

In the ASI neuron, DAF-7/TGF- $\beta$ pathway components regulate behaviors that are directly dependent on the sensory capabilities of the animals and display appropriate physiological behaviors like aggregation, dauer formation, etc. (Ren et al., 1996; Riddle and Albert, 1997; Peckol et al., 2001; Nolan et al., 2002; Gallagher et al., 2013). It has been reported previously that in the absence of exp-1, daf-7 levels are also reduced (Bendesky et al., 2012). We tested the involvement of daf-7 in exp-1 function by performing chemotaxis assays using the exp-1; daf-7 double mutants. Our results showed that daf-7 mutants are not defective in their response toward AWC-dependent odors. However, daf-7 mutations completely suppressed the exp-1 mutant phenotype and the double mutants behaved like WT control animals (Fig. 4A). Next, we asked how daf-7 loss might be suppressing the exp-1 phenotype. One possibility was change in expression of GPCRs 
A

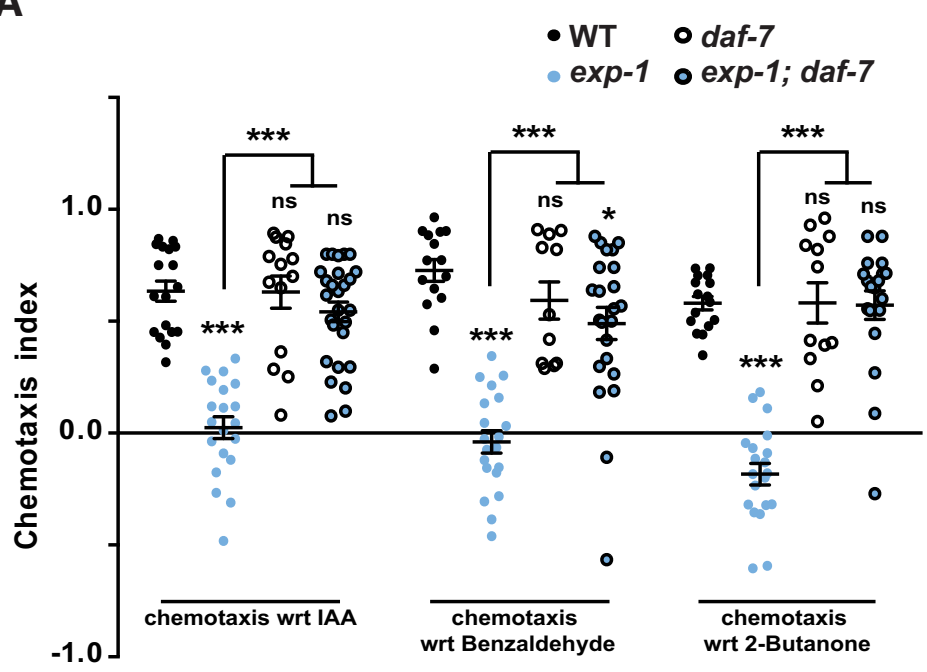

B
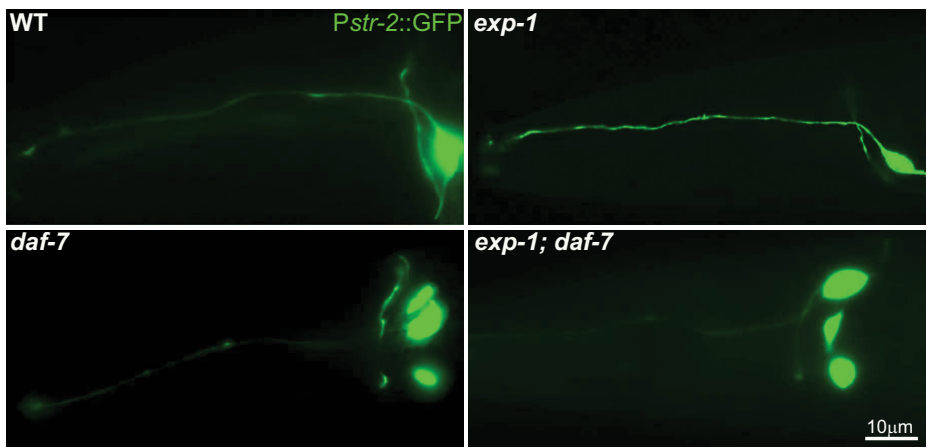

C

- WT $\circ$ str-2

- exp-1 exp-1; str-2

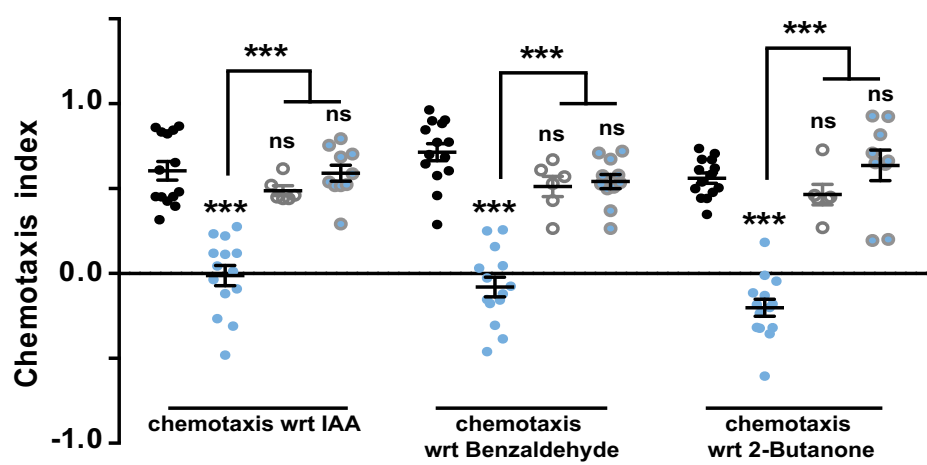

Figure 4. Mutants in daf-7 suppress the exp-1 mutant phenotype. $\boldsymbol{A}$, Graph indicating chemotaxis indices of exp-1; daf-7 animals along with control C. elegans. B, Pstr-2::GFP expression in exp-1; daf-7 mutants along with WT and single mutant animals. $\boldsymbol{C}$, Graph indicating chemotaxis indices of exp-1; str-2 C. elegans along with control animals. Each circle in graphs $\boldsymbol{A}$, $\boldsymbol{C}$ represents one assay performed using $\sim 200-250$ C. elegans. Error bars represent SEM; $p$ values are indicated as ${ }^{* *} p<0.001$, ${ }^{*} p<0.05$; ns, not significant in all graphs based on $p$ values calculated using one-way ANOVA with Bonferroni multiple comparison.

regulated by DAF-7/TGF- $\beta$, present on the cilia for the detection of odors (Nolan et al., 2002). STR-2 is a GPCR serpentine receptor, normally expressed in one of the two AWC neurons and shows low/no expression in ASI in WT conditions. STR-2 expression in the ASI neuron is increased in the absence of daf-7 or during conditions that result in ASI-dependent behaviors (Peckol et al., 2001; Nolan et al., 2002). Expression shift of STR-2 is interesting since no receptor related function is reported for this developmental shift. It has been shown that DAF-7/TGF- $\beta$ genetically interacts with EXP-1 (Bendesky et al., 2012). To probe the possibility that loss of exp-1 might also be 
able to affect the STR-2 expression pattern like DAF-7/ TGF- $\beta$, we examined the expression pattern of STR-2 in the exp-1 mutant background. Our imaging results revealed that exp-1 mutants did not show obvious defects in STR-2::GFP expression, it was expressed unilaterally in the AWC neuron similar to what was seen in WT control animals (Fig. 4B, upper panels). As reported previously, we also found that daf-7 mutants showed upregulated expression of STR-2 in the ASI neuron (Fig. 4B, lower left panel). Our imaging results for exp-1; daf-7 double mutants showed that here too the expression pattern was altered for STR-2, and it was expressed in both the ASI and AWC neurons similar to what was seen in daf-7 mutants (Fig. 4B, lower right panel). These data suggested that exp-1 does not affect the STR-2 expression levels in the ASI neurons. We next went on to study the role of str-2 mutants in conjunction with exp-1. We tested str-2 mutant animals in chemotaxis assays and found that they were not defective in chemotaxis toward AWC-sensed odors. Next, we tested the exp-1; str-2 double mutants and found that these animals behaved like str-2 single mutants (Fig. 4C). Thus, the exp-1 defects toward AWCsensed odors require a functionally WT form of STR-2 which is likely to function as a receptor as previously shown for 2-heptanone (Zhang et al., 2016). We also investigated whether STR-2 was functioning in ASI or AWC neurons to participate in exp-1 mutant chemosensory defects by using the gpa- 4 and str-2 endogenous promoters to drive STR-2 expression in the ASI and AWC neurons, respectively. These rescue constructs were transformed into exp-1; str-2 double mutants and studied for their chemotaxis responses Quantitative analysis of chemotaxis revealed that STR-2 when expressed in the AWC but not in the ASI neurons could partially recapitulate the exp-1 mutant behaviors (Extended Data Fig. 4-1A,B). These results indicate that the exp-1 mutant behavior is dependent on ASI secreted DAF-7 and AWC located STR-2.

\section{STR-2 and EXP-1 also regulate dauer formation}

Based on previous work and our studies it is evident that EXP-1 and STR-2 function in ASI amphid neurons and can regulate behaviors through DAF-7/TGF- $\beta$. Both ASI neurons and DAF-7/TGF- $\beta$ affect dauer formation (Thomas et al., 1993; Peckol et al., 2001; Cheung et al., 2004; Shaw et al., 2007). This got us interested in studying the role of STR-2 and EXP-1 in dauer formation. To test this, we performed dauer assays using exp-1, daf-7, and str-2 mutants as controls along with their double and triple mutants, at temperatures of 16,20 and $25^{\circ} \mathrm{C}$. We observed that str-2 mutant animals did not show differences in the percentage of dauers when compared with WT animals at $16^{\circ} \mathrm{C}$, but dauer formation was slightly, albeit significantly higher at $20^{\circ} \mathrm{C}$. Next, we generated daf-7; str-2 double mutants, that were difficult to obtain and involved screening through hundreds of heterozygous animals. Surprisingly, the double mutants showed significant increase in dauers when compared with either of the two single mutant animals at $16^{\circ} \mathrm{C}$ and $20^{\circ} \mathrm{C}$ (Fig. 5B). Thus, our dauer experiments reveal that STR-2 might be responding to changes in environmental conditions in the absence of daf-7 through alternate pathways. However, double mutants of daf-7; str-2 are not completely dauer, suggesting that there could be molecules still sensing environmental signals in these mutant animals. Since exp-1 is also a receptor, we explored the possibility of its role in sensing the environment and in maintaining the reproductive phase versus entry into the dormant dauer stage. Dauer quantitation showed that exp-1 mutants by themselves behaved like WT control animals and exp-1; daf-7 double mutants behaved like daf-7 mutants. We also tested double mutants of exp-1; str-2 and found that they showed a small but significant increase in the dauer phenotype at $20^{\circ} \mathrm{C}$. Next, we generated triple mutants where exp-1, daf-7, and str-2 were simultaneously removed. Surprisingly, we found that these animals showed close to $80 \%$ dauer phenotype even at $16^{\circ} \mathrm{C}$ and the severity increased to close to $100 \%$ dauers at higher temperatures. Given that EXP-1 and STR-2 are receptors are expressed in ASI and the increased dauer phenotype in the triple mutants was evident even at lower growth temperatures and in the presence of food, a plausible explanation for these results could be that EXP-1 and STR-2 sense environmental signals and modulate developmental balance toward reproductive phase through alternate pathway/s in the absence of DAF-7/TGF- $\beta$.

\section{STR-2 expression in ASI maintains reproductive growth in the absence of daf-7}

Our data so far suggest that STR-2 contributes to maintain reproductive growth phase in the absence of daf-7. Since in daf-7 mutants, STR-2 expression increases in the ASI neuron, thus it is appropriate to assume that it acts as a receptor in this neuron for the perception of conditions favoring reproductive state over the dauer state. To confirm the function of STR-2 in the ASI neuron for dauer formation, we used the gpa-4 promoter and expressed STR2 (Pgpa-4::str-2) in the ASI neuron. We transformed this construct in the dauer forming double (daf-7; str-2) and triple (exp-1; daf-7; str-2) mutant backgrounds. These dauer-forming animals showed a large recovery on STR-2 expression in the ASI neuron and appeared to behave like WT animals (Fig. $6 A, B$ ). These data further supported our hypothesis that STR-2 can function in ASI neuron by activating unknown alternate pathway/s. These results also suggest that in the absence of daf-7, STR-2 allows for sensing favorable conditions such as food.

The dauer phenotypes observed with daf-7; str-2 double and exp-1; daf-7; str-2 triple mutants were obvious even at $16^{\circ} \mathrm{C}$ in the presence of food, implying that sensory loss of olfaction might be the reason for this developmental defect. These findings further indicated that STR-2 and EXP-1 sense food cues in ASI neurons and the dauer phenotypes observed for these mutants could be largely because of the sensory defects. Thus, we next tested these double and triple mutants for chemotaxis toward IAA, Benzaldehyde and 2-Butanone. To perform chemotaxis assays, we specifically took the non-dauer animals as dauer animals are completely chemosensation defective (Bargmann and Horvitz, 1991). Non-dauer animals were collected by sedimentation that enriches for non- 
A

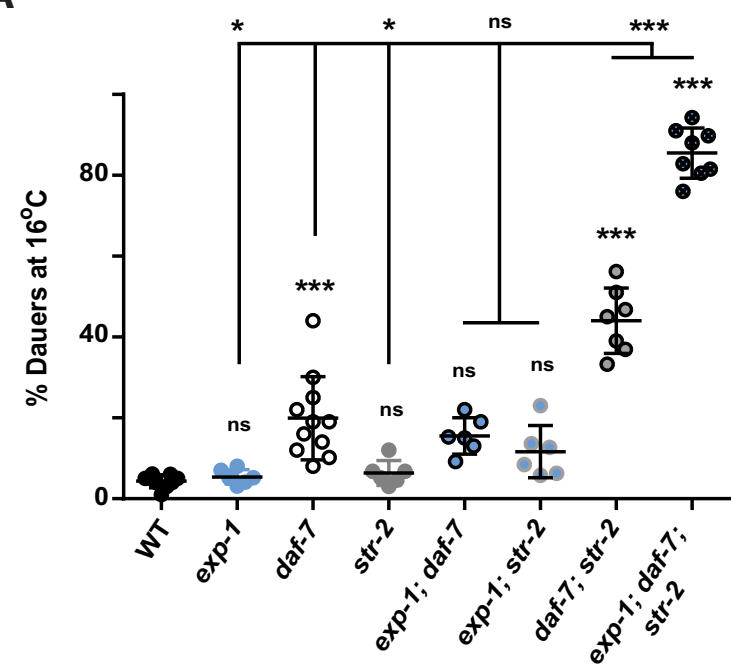

B

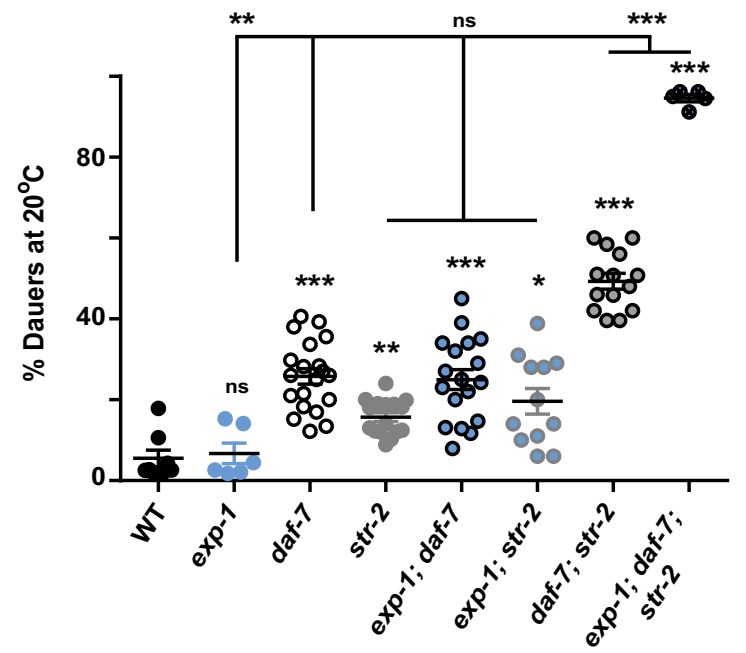

C

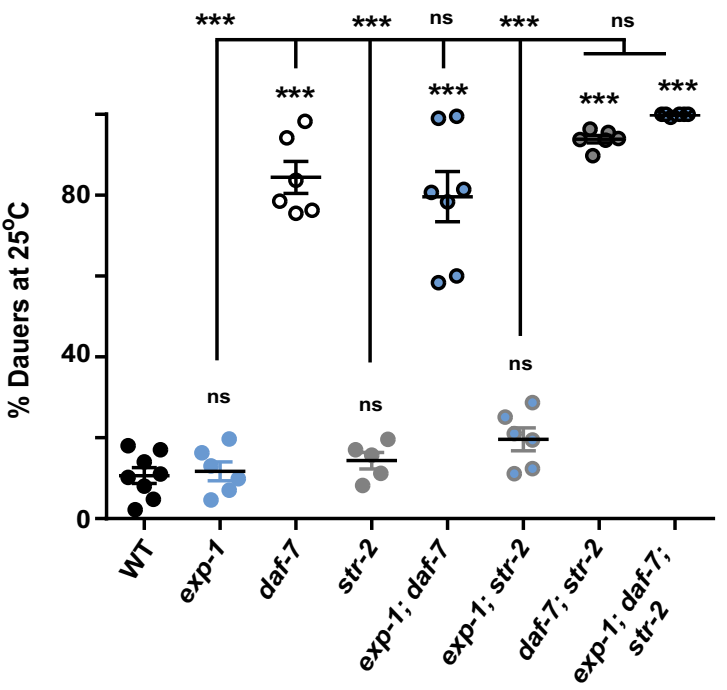

Figure 5. Loss of str-2 in the daf-7 mutant background leads to an enhanced dauer phenotype that is further enhanced in the absence of exp-1. Dauer assays were performed for the exp-1; continued

daf-7; str-2 mutant animals along with WT, single mutant, and double mutant $C$. elegans. The assay was performed at three temperatures: $(\boldsymbol{A}) 16^{\circ} \mathrm{C},(\boldsymbol{B}) 20^{\circ} \mathrm{C}$, and $(\boldsymbol{C}) 25^{\circ} \mathrm{C}$. Each circle in the graphs represents a single experiment performed using 200-300 animals per plate. Error bars represent SEM; $p$ values are indicated as ${ }^{* \star *} p<0.001,{ }^{* \star} p<0.01,{ }^{\star} p<0.05$; ns, not significant in all graphs based on $p$ values calculated using oneway ANOVA with Bonferroni multiple comparison test where relevant.

dauers (heavier animals). The lighter dauer animals were discarded. We found that the double mutants of daf-7; str-2 were also defective in their response to AWC-sensed odors (Fig. $6 C, D$ ). Moreover, we found that the triple mutant dauer animals either did not move or moved toward the control odor. The ASI-specific rescue of STR-2 could modestly overcome the chemotactic defects in these mutants (Fig. 6C,D). In daf-7 mutants, STR-2 is also expressed in AWC neurons along with its upregulation in ASI. Since the odors tested here were mainly detected through the AWC neuron, thus it indicates that chemotaxis defect of str-2 could be because of changes in its expression pattern and also because of changes in animal physiology associated with dauer phenotype (Cassada and Russell, 1975). Taken together, our data suggest that EXP-1 and STR-2 contribute toward the maintenance of the reproductive state during development and may function in the absence of DAF-7/TGF- $\beta$ through other signaling pathways.

\section{TGF- $\beta$ molecule, DBL- 1 also participates in chemosensation and modulates dauer formation}

Previous reports indicate that another TGF- $\beta$ molecule, DBL-1 also prevents entry into dauer phase (Roberts et al., 2010). DBL-1 is expressed in AFD amphid neurons in the head region in addition to other neurons. AFD neurons are temperature-sensing neurons, and it is possible that $\mathrm{DBL}-1$ is regulating the dauer-reproductive switch based on temperature. Studies have shown that fluctuations in DAF-7/TGF- $\beta$ levels are not temperature dependent (O'Donnell et al., 2018). We generated daf-7; $d b /$-1 double mutant and performed dauer assays (Fig. 7A). It was interesting to find that these animals also showed increased percentage in dauer formation compared with the control animals. Since DBL- 1 and DAF-7/TGF- $\beta$ are expressed and released from different cell types, there is a possibility that they initially act in parallel in a distributed neuronal network and maybe finally integrate the signals through their common Type II receptor DAF-4 (Estevez et al., 1993). We also performed chemotaxis experiments using AWC-dependent odors for $d b /-1$ mutants. Our results showed that this double mutant combination of daf-7; $d b /-1$ were also defective for AWC-sensed odors (Fig. 7B) as was the case for other double and triple mutants of daf-7. Data here support our above results that sensory cues from food are coupled to developmental dauer switch mediated through endocrine TGF- $\beta$ pathways (DAF-7 and DBL-1). 
A

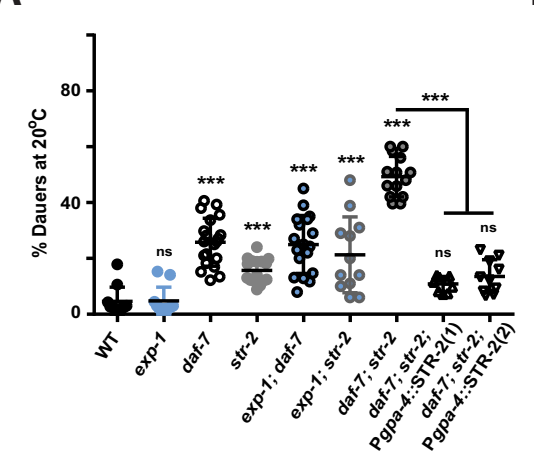

B

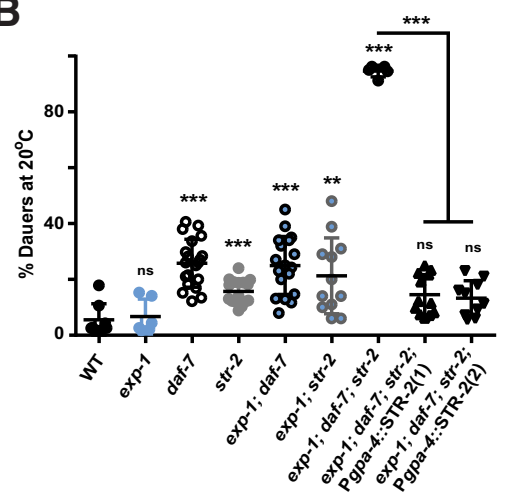

C
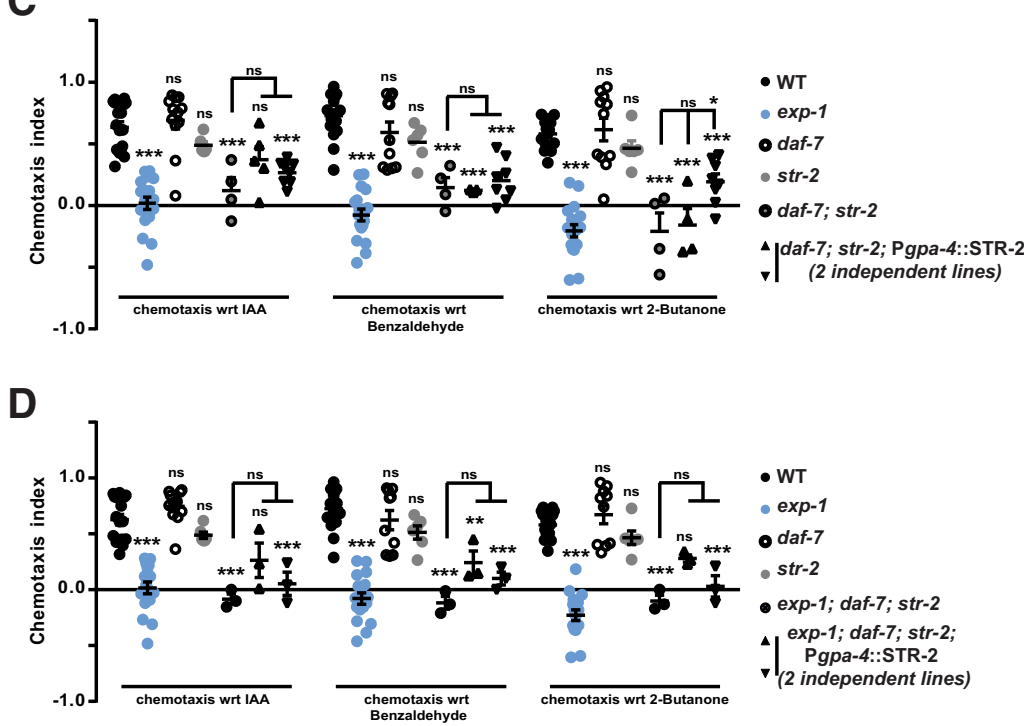

Figure 6. STR-2 expression in ASI neurons completely rescues the dauer phenotype and partially rescues the chemotaxis defects of exp-1; daf-7; str-2 mutant animals. A, STR-2 rescue of the dauer phenotype of daf-7; str-2 mutant animals along with controls. This experiment was performed at $20^{\circ} \mathrm{C}$. $\boldsymbol{B}$, STR-2 rescue of the dauer phenotype of exp-1; daf-7; str-2 mutant animals along with controls. This experiment was performed at $20^{\circ} \mathrm{C}$. $\boldsymbol{A}, \boldsymbol{B}$, Each circle or triangle in the graphs represents a single experiment performed using 200-300 animals per 60-mm plate. C, Graph representing chemotaxis indices for STR-2 rescue assays performed in double mutant (daf-7; str-2) animals along with controls. $\boldsymbol{D}$, Graph representing chemotaxis indices for STR-2 rescue assays performed in triple mutant (exp-1; daf-7; str-2) animals along with controls. Each circle or triangle in graphs $\boldsymbol{C}, \boldsymbol{D}$, represents one assay performed using $\sim 200-250$ C. elegans. Error bars represent SEM; $p$ values are indicated as ${ }^{* * *} p<0.001,{ }^{* *} p<0.01$, ${ }^{*} p<0.05$; ns, not significant in all graphs based on $p$ values calculated using one-way ANOVA with Bonferroni multiple comparison test where relevant.

\section{Discussion}

Our study suggests that in C. elegans, chemosensory neural circuits are flexible and that their neuronal signaling is modified by developmental stages, their environment, the receptors expressed by the neurons and the signaling molecules secreted by them. Previous studies showed that EXP-1, an excitatory GABA receptor functions to modulate physiological behaviors of aggregation and bordering in the presence of food (Bendesky et al., 2012). N2 WT animals aggregate to avoid hyperoxia, population density, and stressful conditions such as aversive odors and food scarcity (de Bono et al., 2002; Chang et al., 2006). Food stimuli are important modulators of social behaviors as mutants prone to aggregation do not show behavior in the absence of food (de Bono et al., 2002). Chemosensory neurons (ASH, ADL, ASI) and their receptors (OCR-2, OSM-9, ODR-4, ODR-10) have been implicated in aggregation behavior (de Bono et al., 2002; Rogers et al., 2006). A study by Bendesky et al. (2012) has shown that social behaviors such as aggregation are dependent on many genes that show quantitative effects on behavior through genetic variations. This study also suggested that multiple receptors and ligand gated ion channels are suitable candidates for genetic variations with respect to their function in social behaviors in both C. elegans and humans (Bendesky et al., 2012). Same study indicated that EXP-1 may participate in detection of environmental signals (food) and hence modulate DAF-7 levels (Bendesky et al., 2012). Our initial experiments were based on the hypothesis that aggregation behavior is indirectly linked to sensory defects in food/attractive odors. Our results correlated with published results and our hypothesis where exp-1 mutants were defective for AWC-dependent attractive odors and behaved normally 
A

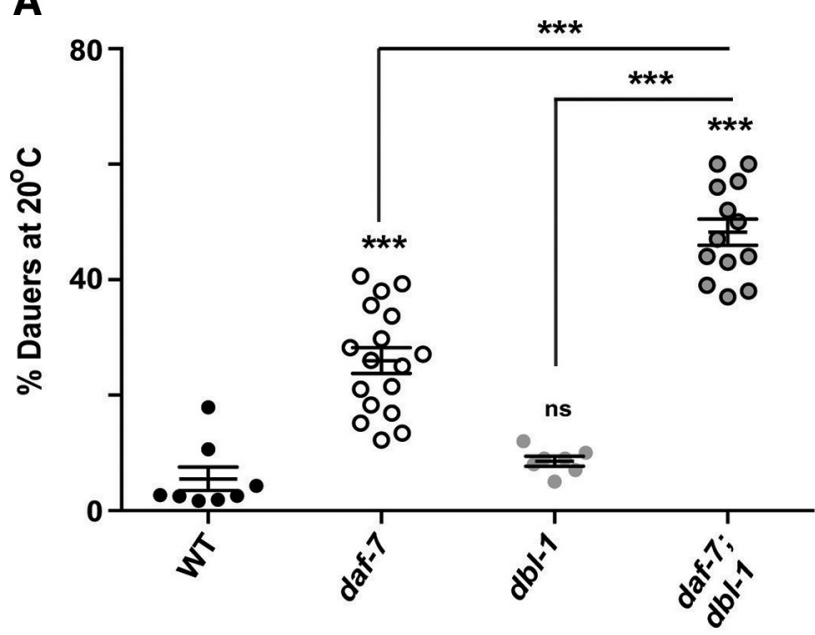

C

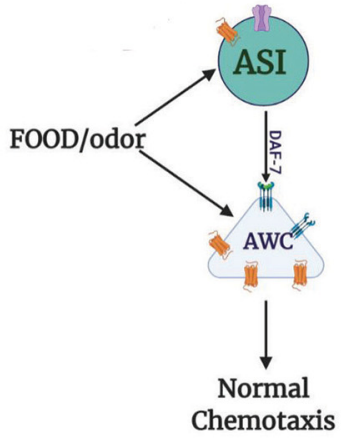

i

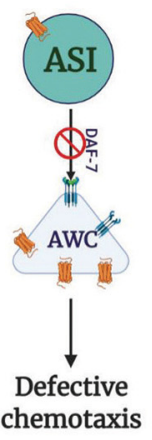

ii
B

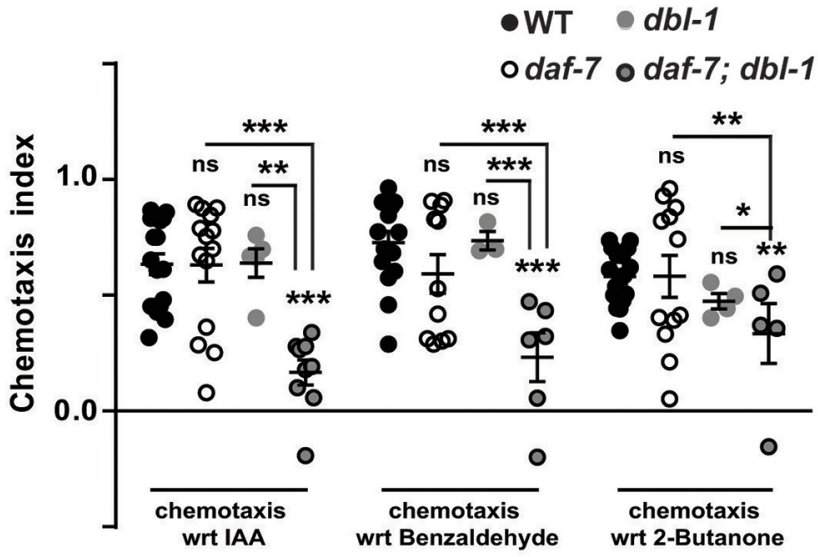

$\int 2$ EXP-1

if STR-2

4if DAF - 1/DaF - 4

$\checkmark$ DAF - 7

DBL- 1

Figure 7. Mutants of $d b l-1$ (TGF $\beta$ /BMP-like ligand) along with daf-7 mutants resulted in dauer as well as chemotaxis defective phenotypes. $\boldsymbol{A}$, Graph indicating the dauer phenotype of daf-7; $d b /-1 \mathrm{C}$. elegans along with control animals. This experiment was performed at $20^{\circ} \mathrm{C}$. Each circle in the graphs represents a single experiment performed using $\sim 200-300$ animals per $60-\mathrm{mm}$ plate. $\boldsymbol{B}$, Graph indicating chemotaxis indices of daf-7; $d b /-1$ C. elegans along with control animals. Each circle in the graphs represents one assay performed using 200-250 C. elegans. Error bars represent SEM; $p$ values are indicated as ${ }^{* * *} p<0.001$, ${ }^{* *} p<0.01$, ${ }^{*} p<0.05$; ns, not significant in all graphs based on $p$ values calculated using one-way ANOVA with Bonferroni multiple comparison test where relevant. $\boldsymbol{C}$, A predicted model for how AWC-dependent and ASI-dependent chemosensory cues could lead to dauer formation. $\boldsymbol{i}$, Normally AWC is responsible for sensing attractive odors and ASI senses food-dependent odors. They communicate with each other and maintain the reproductive phase of the organism. ii, In the absence of exp-1, ASI neuron is able to sense the volatile odors because of retracted cilia. Here, levels of DAF-7 are low (EXP-1 regulates DAF-7 expression levels) and as a result the communication between AWC and ASI is aberrant. Moreover, ASI also has strong connections with downstream interneurons (AIB, AIY, AIA), which are normally involved in AWC chemosensory signaling leading to chemosensory bias between AWC and ASI neuron signaling and hence repulsion toward attractive odors. iii, In the absence of daf-7, STR-2 expression in the ASI neuron is upregulated and our results indicate that both STR-2 and EXP-1 might be sensing food odors and communicating with the AWC neuron through an alternate pathway (possibly DBL-1 dependent). This could explain why double mutants of daf-7 with str-2, exp-1, and $d b /-1$ all show increased percentage of dauers as compared with daf-7 single mutants. Moreover, triple mutants of daf-7 with both str-2 and exp-1 mutations show an exaggerated dauer phenotype, possibly because of the loss of the alternate food sensory pathway (dashed line) in the absence of daf-7.

toward odors sensed by other amphid neurons (AWA and AWB). In later experiments, our expression studies revealed that contrary to our expectation and its phenotypes, EXP-1 was not expressed in the AWC neurons but showed expression in the ASI neurons. The ASI neuron is also a chemosensory amphid neuron and modulates various behaviors in response to diverse environmental cues via DAF-7/TGF- $\beta$ and other neurohormones released by it. Additional experiments showed that the chemotaxis defects were ASI neuron dependent and DAF-7/TGF- $\beta$ was necessary for this defect.
The DAF-7/TGF- $\beta$ pathway is a major regulator of dauer formation, an alternative growth stage. The dauer animals withdraw the ciliated endings from the amphid pore that is accompanied by changes in spatial organization of receptor proteins (SRD-1, STR-2, and STR-3; Lans and Jansen, 2006). Studies in the past have revealed that misexpression of proteins such as receptors and transcription factors can result in neuronal defects and altered functions (Sagasti et al., 1999). STR-2 is normally expressed in the AWC neuron but during adverse environmental 
conditions or in the absence of the DAF-7/TGF- $\beta$ pathway it was strongly upregulated in the ASI neurons (Nolan et al., 2002). Direct correlation between ASI-specific expression of STR-2-dependent and dauer-dependent ASI cilia retraction was observed (Peckol et al., 2001). Neurons with retracted cilia can detect volatile odors (Ward et al., 1975; Bargmann et al., 1993; Peckol et al., 2001). Hence, STR-2 on expression in ASI neurons might be able to sense volatile odors. We also found that in case of exp-1 mutants ASI ciliary endings were retracted and thus ASI might be able to sense volatile odors. However, exp-1 mutants show defects toward all odors sensed by the AWC neuron, and there could be various reasons for this. First, it could be because of downstream synaptic connectivity of the sensory neuron. It is possible that ASI could elicit different responses to AWC-sensed attractants because AWC and ASI have different synaptic connections and release nonoverlapping molecules that could activate distinct circuits (White et al., 1986). Second, there could also be defect/s in the AWC neuron sensory ability because of changes at the ciliary receptive ends and the axon shape. In exp-1 mutants, although AWC neuron cilia and axon shape was not obviously defective, it is possible that there are minor defects that we were unable to observe, that could alter the $C$. elegans response toward AWC-perceived odors. However, we found that loss of functional AWC neurons reproduced the exp-1 chemotaxis defects. Thus, there might be a defect in AWC neurons in exp-1 mutants which we could not detect. Third, we believe that animals with metabolic disorders could be defective toward sensory perception of food as has been shown previously (Fujiwara et al., 2002; Mak et al., 2006). Mutants in exp-1 are defective in defecation and are thought to manifest a form of constipation (Beg and Jorgensen, 2003). Thus, it is also possible that because of defective metabolism they develop aversion to food related odors, and this could also be the reason for their increased aggregation and bordering on the sides of food during feeding.

Lowered expression of DAF-7/TGF- $\beta$ is a significant marker of the dauer stage and exp-1 mutants also decrease DAF-7 expression (Murakami et al., 2001). Here, we found that exp-1 mutants were not dauer but exp-1; daf-7 double mutant animals showed increased percentage of dauer animals similar to daf-7 mutants. Thus, DAF7 is epistatic to EXP-1 and may function downstream of EXP-1, an excitatory GABA receptor. Neuronal receptors/ GPCRs are at the interface between the external milieu and the neuron. In a recent study, it was shown that STR2 regulates life span in an ASI-dependent manner (Dixit et al., 2020), and it is well known that dauer animals can live for months as compared with reproductive animals that live for just a few weeks (Cassada and Russell, 1975). STR-2 expression levels are specifically increased in the ASI neuron during dauer like conditions (Lans and Jansen, 2006). This prompted us to investigate the physiological condition of the animal on removal of str-2 from dauer forming animals such as daf-7 mutants. Our data with these double mutants revealed that STR-2 is deployed in the ASI neuron to maintain the reproductive growth phase in the absence of daf-7 or during other dauer inducing conditions such as food scarcity. Chemotaxis results indicate that EXP-1 functions upstream of DAF-7/TGF- $\beta$ and EXP-1 also regulates DAF-7 levels (Bendesky et al., 2012). We reasoned that EXP-1 might also be contributing toward dauer formation. When the exp-1 mutation was introduced in the daf-7; str-2 mutant background, dauer formation increased to very high levels $(\sim 80-100 \%)$ even at conducive growth temperatures of $16^{\circ} \mathrm{C}$ in the presence of food. Perhaps, both EXP-1 and STR-2 receptors are working in the ASI neurons to sense the environment (food) and contribute to the larval fate of the organism. The percentage of dauers increased with the loss of receptors (exp-1; str-2) as compared with daf-7 mutants alone, indicating that the signals received here can also activate other signaling pathways in ASI. This is also supported by our rescue experiment where STR-2 expression in ASI neuron using extrachromosomal arrays brought down the percentage of dauers to WT levels since STR-2 overexpression might bypass daf- 7 loss and function through other signaling pathways to maintain normal growth. To address this idea, we analyzed another TGF- $\beta$ protein DBL- 1 and found that its deletion along with daf-7 loss increased the dauer formation like daf-7; str-2 and exp-1; daf-7; str-2 mutants and showed chemosensory defects. Hence DBL-1 could be functioning in the absence of daf- 7 to maintain reproductive state along with EXP-1 and STR-2.

In this study, we have discovered that exp-1 mutants are defective toward AWC-sensed odors. Furthermore, we have shown expression of EXP-1 in the ASI neuron which could explain the role of EXP-1 in DAF-7-dependent behaviors. We were able to identify new molecules participating in dauer formation and speculate that more genes/pathways are involved in the reproductive-dauer transition switch that could be detected in a sensitized background (summarized as model in Fig. 7C). Our study concludes that food derived attractive chemosensory cues can also be detected by ASI amphid neurons and this in turn could decide the developmental fate of the animal.

\section{References}

Ailion M, Thomas JH (2000) Dauer formation induced by high temperatures in Caenorhabditis elegans. Genetics 156:1047-1067.

Albert PS, Brown SJ, Riddle DL (1981) Sensory control of dauer larva formation in Caenorhabditis elegans. J Comp Neurol 198:435451.

Bargmann Cl (1998) Neurobiology of the Caenorhabditis elegans genome. Science 282:2028-2033.

Bargmann Cl, Horvitz HR (1991) Chemosensory neurons with overlapping functions direct chemotaxis to multiple chemicals in C. elegans. Neuron 7:729-742.

Bargmann Cl, Mori I (1997) Chemotaxis and thermotaxis. In: C. elegans II (Riddle DL, Blumenthal T, Meyer BJ, Priess JR, eds). New York: Cold Spring Harbor Laboratory Press.

Bargmann Cl, Hartwieg E, Horvitz HR (1993) Odorant-selective genes and neurons mediate olfaction in C. elegans. Cell 74:515527.

Beg AA, Jorgensen EM (2003) EXP-1 is an excitatory GABA-gated cation channel. Nat Neurosci 6:1145-1152.

Bendesky A, Pitts J, Rockman MV, Chen WC, Tan MW, Kruglyak L, Bargmann $\mathrm{Cl}$ (2012) Long-range regulatory polymorphisms affecting a GABA receptor constitute a quantitative trait locus (QTL) for 
social behavior in Caenorhabditis elegans. PLoS Genet 8: e1003157.

Birnby DA, Link EM, Vowels JJ, Tian H, Colacurcio PL, Thomas JH (2000) A transmembrane guanylyl cyclase (DAF-11) and Hsp90 (DAF-21) regulate a common set of chemosensory behaviors in Caenorhabditis elegans. Genetics 155:85-104.

Brenner S (1974) The genetics of Caenorhabditis elegans. Genetics 77:71-94.

Cassada RC, Russell RL (1975) The dauerlarva, a post-embryonic developmental variant of the nematode Caenorhabditis elegans. Dev Biol 46:326-342.

Chalasani SH, Chronis N, Tsunozaki M, Gray JM, Ramot D, Goodman MB, Bargmann Cl (2007) Dissecting a circuit for olfactory behaviour in Caenorhabditis elegans. Nature 450:63-70.

Chang AJ, Chronis N, Karow DS, Marletta MA, Bargmann Cl (2006) A distributed chemosensory circuit for oxygen preference in $C$. elegans. PLoS Biol 4:e274.

Chao MY, Komatsu H, Fukuto HS, Dionne HM, Hart AC (2004) Feeding status and serotonin rapidly and reversibly modulate a Caenorhabditis elegans chemosensory circuit. Proc Natl Acad Sci USA 101:15512-15517.

Cheung BH, Arellano-Carbajal F, Rybicki I, de Bono M (2004) Soluble guanylate cyclases act in neurons exposed to the body fluid to promote C. elegans aggregation behavior. Curr Biol 14:11051111.

Cho SW, Choi KY, Park CS (2004) A new putative cyclic nucleotidegated channel gene, cng-3, is critical for thermotolerance in Caenorhabditis elegans. Biochem Biophys Res Commun 325:525531.

Cho SW, Cho JH, Song HO, Park CS (2005) Identification and characterization of a putative cyclic nucleotide-gated channel, CNG-1, in. C. elegans. Mol Cells 19:149-154.

Coburn CM, Bargmann Cl (1996) A putative cyclic nucleotide-gated channel is required for sensory development and function in C. elegans. Neuron 17:695-706.

da Graca LS, Zimmerman KK, Mitchell MC, Kozhan-Gorodetska M, Sekiewicz K, Morales Y, Patterson GI (2004) DAF-5 is a Ski oncoprotein homolog that functions in a neuronal TGF beta pathway to regulate C. elegans dauer development. Development 131:435446.

Dahiya Y, Rose S, Thapliyal S, Bhardwaj S, Prasad M, Babu K (2019) Differential regulation of innate and learned behavior by Creb1/ Crh-1 in Caenorhabditis elegans. J Neurosci 39:7934-7946.

Daniels SA, Ailion M, Thomas JH, Sengupta P (2000) egl-4 acts through a transforming growth factor-beta/SMAD pathway in Caenorhabditis elegans to regulate multiple neuronal circuits in response to sensory cues. Genetics 156:123-141.

de Bono M, Tobin DM, Davis MW, Avery L, Bargmann Cl (2002) Social feeding in Caenorhabditis elegans is induced by neurons that detect aversive stimuli. Nature 419:899-903.

Dixit A, Sandhu A, Modi S, Shashikanth M, Koushika SP, Watts JL, Singh V (2020) Neuronal control of lipid metabolism by STR-2 G protein-coupled receptor promotes longevity in Caenorhabditis elegans. Aging Cell 19:e13160.

Estevez M, Attisano L, Wrana JL, Albert PS, Massagué J, Riddle DL (1993) The daf-4 gene encodes a bone morphogenetic protein receptor controlling $C$. elegans dauer larva development. Nature 365:644-649.

Fujiwara M, Sengupta P, McIntire SL (2002) Regulation of body size and behavioral state of $C$. elegans by sensory perception and the EGL-4 cGMP-dependent protein kinase. Neuron 36:1091-1102.

Gallagher T, Kim J, Oldenbroek M, Kerr R, You YJ (2013) ASI regulates satiety quiescence in C. elegans. J Neurosci 33:9716-9724.

Golden JW, Riddle DL (1982) A pheromone influences larval development in the nematode Caenorhabditis elegans. Science 218:578580.

Golden JW, Riddle DL (1984a) The Caenorhabditis elegans dauer larva: developmental effects of pheromone, food, and temperature. Dev Biol 102:368-378.
Golden JW, Riddle DL (1984b) A pheromone-induced developmental switch in Caenorhabditis elegans: temperature-sensitive mutants reveal a wild-type temperature-dependent process. Proc Natl Acad Sci USA 81:819-823.

Goldmakher GV, Moss RL (2000) A subset of periglomerular neurons in the rat accessory olfactory bulb may be excited by GABA through a $\mathrm{Na}(+)$-dependent mechanism. Brain Res 871:7-15.

Gottlieb S, Ruvkun G (1994) daf-2, daf-16 and daf-23: genetically interacting genes controlling Dauer formation in Caenorhabditis elegans. Genetics 137:107-120.

Gunther CV, Georgi LL, Riddle DL (2000) A Caenorhabditis elegans type I TGF beta receptor can function in the absence of type II kinase to promote larval development. Development 127:33373347.

Hart AC, Chao MY (2010) From odors to behaviors in Caenorhabditis elegans. In: The neurobiology of olfaction (Menini A, ed). Boca Raton: CRC Press/Taylor \& Francis.

Inoue T, Thomas JH (2000) Targets of TGF-beta signaling in Caenorhabditis elegans dauer formation. Dev Biol 217:192-204.

Kadam NY, Behera S, Kumar S, Ghosh-Roy A, Babu K (2021) The Gprotein-coupled receptor SRX-97 is required for concentration-dependent sensing of benzaldehyde in caenorhabditis elegans. eNeuro 8(1):ENEURO.0011-20.2020.

Komatsu $\mathrm{H}$, Jin YH, L'Etoile N, Mori I, Bargmann Cl, Akaike N, Ohshima Y (1999) Functional reconstitution of a heteromeric cyclic nucleotide-gated channel of Caenorhabditis elegans in cultured cells. Brain Res 821:160-168.

Lanjuin A, VanHoven MK, Bargmann CI, Thompson JK, Sengupta P (2003) Otx/otd homeobox genes specify distinct sensory neuron identities in C. elegans. Dev Cell 5:621-633.

Lans H, Jansen G (2006) Noncell- and cell-autonomous G-protein-signaling converges with $\mathrm{Ca} 2+/$ mitogen-activated protein kinase signaling to regulate str-2 receptor gene expression in Caenorhabditis elegans. Genetics 173:1287-1299.

Mak HY, Nelson LS, Basson M, Johnson CD, Ruvkun G (2006) Polygenic control of Caenorhabditis elegans fat storage. Nat Genet 38:363-368.

Margie O, Palmer C, Chin-Sang I (2013) C. elegans chemotaxis assay. J Vis Exp. Advance online publication. Retrieved April 27, 2013. doi: $10.3791 / 50069$.

Mello C, Fire A (1995) DNA transformation. Methods Cell Biol 48:451-482.

Mello CC, Kramer JM, Stinchcomb D, Ambros V (1991) Efficient gene transfer in C.elegans: extrachromosomal maintenance and integration of transforming sequences. EMBO J 10:3959-3970.

Murakami M, Koga M, Ohshima Y (2001) DAF-7/TGF-beta expression required for the normal larval development in $C$. elegans is controlled by a presumed guanylyl cyclase DAF-11. Mech Dev 109:27-35.

Nolan KM, Sarafi-Reinach TR, Horne JG, Saffer AM, Sengupta P (2002) The DAF-7 TGF-beta signaling pathway regulates chemosensory receptor gene expression in C. elegans. Genes Dev 16:3061-3073.

O'Donnell MP, Chao PH, Kammenga JE, Sengupta P (2018) Rictor/ TORC2 mediates gut-to-brain signaling in the regulation of phenotypic plasticity in C. elegans. PLoS Genet 14:e1007213.

O'Halloran DM, Altshuler-Keylin S, Lee JI, L'Etoile ND (2009) Regulators of AWC-mediated olfactory plasticity in Caenorhabditis elegans. PLoS Genet 5:e1000761.

Oshio K, Iwasaki Y, Morita S, Osana Y, Gomi S, Akiyama E, Omata K, Oka K, Kawamura K (2003) Database of synaptic connectivity of C. elegans for computation. Technical Report of CCeP, Keio Future, No.1, Keio University.

Patterson GI, Koweek A, Wong A, Liu Y, Ruvkun G (1997) The DAF-3 Smad protein antagonizes TGF-beta-related receptor signaling in the Caenorhabditis elegans dauer pathway. Genes Dev 11:26792690.

Peckol EL, Troemel ER, Bargmann Cl (2001) Sensory experience and sensory activity regulate chemosensory receptor gene expression in Caenorhabditis elegans. Proc Natl Acad Sci USA 98:11032-11038. 
Ren P, Lim CS, Johnsen R, Albert PS, Pilgrim D, Riddle DL (1996) Control of $C$. elegans larval development by neuronal expression of a TGF-beta homolog. Science 274:1389-1391.

Riddle DL, Albert PS (1997) Genetic and environmental regulation of dauer larva development. In: C. elegans II (Riddle DL, Blumenthal T, Meyer BJ, Priess JR, eds). New York: Cold Spring Harbor Laboratory Press.

Roberts AF, Gumienny TL, Gleason RJ, Wang H, Padgett RW (2010) Regulation of genes affecting body size and innate immunity by the DBL-1/BMP-like pathway in Caenorhabditis elegans. BMC Dev Biol 10:61.

Rogers C, Persson A, Cheung B, de Bono M (2006) Behavioral motifs and neural pathways coordinating $\mathrm{O} 2$ responses and aggregation in C. elegans. Curr Biol 16:649-659.

Russell JS (2001) Molecular cloning: a laboratory manual. New York: Cold Spring Harbor Laboratory Press.

Sagasti A, Hobert O, Troemel ER, Ruvkun G, Bargmann CI (1999) Alternative olfactory neuron fates are specified by the LIM homeobox gene lim-4. Genes Dev 13:1794-1806.

Schackwitz WS, Inoue T, Thomas JH (1996) Chemosensory neurons function in parallel to mediate a pheromone response in C. elegans. Neuron 17:719-728.

Schindelin J, Arganda-Carreras I, Frise E, Kaynig V, Longair M, Pietzsch T, Preibisch S, Rueden C, Saalfeld S, Schmid B, Tinevez JY, White DJ, Hartenstein V, Eliceiri K, Tomancak P, Cardona A (2012) Fiji: an open-source platform for biological-image analysis. Nat Methods 9:676-682.

Sengupta P (2007) Generation and modulation of chemosensory behaviors in C. elegans. Pflugers Arch 454:721-734.

Sengupta P, Chou JH, Bargmann Cl (1996) odr-10 encodes a seven transmembrane domain olfactory receptor required for responses to the odorant diacetyl. Cell 84:899-909.
Shaw WM, Luo S, Landis J, Ashraf J, Murphy CT (2007) The C. elegans TGF-beta Dauer pathway regulates longevity via insulin signaling. Curr Biol 17:1635-1645.

Sieburth D, Ch'ng QLim, Dybbs M, Tavazoie M, Kennedy S, Wang D, Dupuy D, Rual J-F, Hill DE, Vidal M, Ruvkun G, Kaplan JM (2005) Systematic analysis of genes required for synapse structure and function. Nature 436:510-517.

Thomas JH, Birnby DA, Vowels JJ (1993) Evidence for parallel processing of sensory information controlling dauer formation in Caenorhabditis elegans. Genetics 134:1105-1117.

Troemel ER, Chou JH, Dwyer ND, Colbert HA, Bargmann Cl (1995) Divergent seven transmembrane receptors are candidate chemosensory receptors in C. elegans. Cell 83:207-218.

Vowels JJ, Thomas JH (1994) Multiple chemosensory defects in daf11 and daf-21 mutants of Caenorhabditis elegans. Genetics 138:303-316.

Ward S, Thomson N, White JG, Brenner S (1975) Electron microscopical reconstruction of the anterior sensory anatomy of the nematode Caenorhabditis elegans.?2UU. J Comp Neurol 160:313-337.

White JG, Southgate E, Thomson JN, Brenner S (1986) The structure of the nervous system of the nematode Caenorhabditis elegans. Philos Trans R Soc Lond B Biol Sci 314:1-340.

Zhang C, Zhao N, Chen Y, Zhang D, Yan J, Zou W, Zhang K, Huang X (2016) The signaling pathway of Caenorhabditis elegans mediates chemotaxis response to the attractant 2-heptanone in a Trojan horse-like pathogenesis. J Biol Chem 291: 23618-23627.

Zwaal RR, Mendel JE, Sternberg PW, Plasterk RH (1997) Two neuronal $G$ proteins are involved in chemosensation of the Caenorhabditis elegans Dauer-inducing pheromone. Genetics 145:715-727. 